\title{
Reception of Skywave Signals Near a Coastline
}

\author{
J. Bach Andersen
}

Contribution from Laboratory of Electromagnetic Theory, The Technical University of Denmark, Copenhagen, Denmark

(Received November 5, 1962)

\begin{abstract}
An experimental investigation has been made on the influence of ground inhomogeneities on the reception of skywave signals, especially the influence of the conductivity contrast near a coastline. This gives rise to a rapid decrease in field strength near the coastline as is well known from groundwave mixed path theory. Comparison with theory is given. Influence of diffuse reflection from the ionosphere is also considered.
\end{abstract}

\section{Introduction}

Many factors influence the radiation or reception of skywave signals in the shortwave range, and in choosing a proper site for a HF-antenna it is important to evaluate the influence of the various factors. They include the electrical parameters (the conductivity and the permittivity) of the ground, hills, or cliffs and other irregularities of the shape of the ground surface, and the curvature of the earth. It is necessary to take into account that the electrical parameters may vary along the surface of the ground - this is, for example, the case at a coastline and also that they may vary with the depth. This paper deals with the influence of the ground on the reception of skywave signals at an actual site for a receiver station close to the seashore at Reersø, Denmark.

Part of the antenna station which will be erected at Reers $\emptyset$ will receive skywave signals from Godthåb in Greenland. In the day time the ionospheric reflections will take place in the E-layer and during the night in the F-layer, a two-hop path via the E-layer and a one-hop path via the $F$-layer; the geometry is such that the angle of arrival in both cases is very small, about $4^{\circ}$. It is well known that it is difficult to make antennas which operate well at such small angles due to the absorption in the ground.

One method of obtaining an improvement in the reception at low angles is to use an extended groundwire system in the direction of propagation (or reception) [A. C. Wilson, 1961]. Another method would be to choose the antenna site near a coastline in order to utilize the high conductivity of sea water wherever it is possible. However, the antenna would still be situated on a lossy ground and it would be of interest to know the influence of the various sections on the radiation pattern. A theoretical solution to this problem has previously been given [Andersen, 1962] but due to the idealized conditions, i.e., a straight boundary line, flat and homogeneous sections, it was considered worthwhile to measure the field intensity at an actual reception site in order to find the deviations from the simple theory.

Instead of measuring the vertical radiation pattern at different distances from the coastline, the field intensity from a distant transmitter was measured simultaneously at two different places. In this way the relative field strength could be measured as a function of the distance from the coastline, and also the effect of the ionosphere could be accounted for.

To make the study as complete as possible the corresponding curve for the groundwave mixed path was also measured, the transmitter being situated at ground level far from the coastline over the sea. Finally the attenuation of the groundwave on the homogeneous ground was measured in order to find the degree of inhomogeneity of the ground and to estimate the value of the ground constants.

The effect of diffuse reflection from the ionosphere and the effect of the curvature of the earth is also mentioned.

\section{Theory for a Two Section Ground}

In a previous paper [Andersen, 1962] a solution was given for the radiation field from a vertical dipole situated on an inhomogeneous ground consisting of two or three sections with arbitrary electrical properties. ${ }^{1}$ Numerical calculations were given in the case where one or two sections were perfectly conducting. The result for a two section ground is given here for reference where a time factor $\exp (-i \omega t)$ is employed. $A\left(\psi, r_{0}\right)$ is the radiation pattern for a vertical dipole placed on section 1 at a distance $r_{0}$ from section 2 , and $\psi$ is the angle of elevation.

${ }^{1}$ A more general solution has been given by Wait [1961 for the case of a spherical earth. However, it is not in as convenient form as (1) here. 


$$
\begin{aligned}
A=\frac{1+R_{v}^{(1)}}{2} \cos \psi+\frac{i\left(u_{2}-u_{1}\right)}{u_{1}} \frac{1+R_{v}^{(2)}}{2}\left[\frac{\sqrt{2 c}}{c+i}\left(\frac{\sqrt{2}}{2} e^{i \frac{\pi}{4}}-F\left(t_{0}\right)\right)-\frac{i e^{i t}}{(c+i) \sqrt{\pi p_{0}}}\left(1-G\left(p_{0}\right)\right)\right] \\
u_{n}=\sqrt{\frac{\epsilon_{0}}{\epsilon_{n}+i \frac{\sigma_{n}}{\omega}} \quad(n \text {th medium) }} \\
c=\frac{2(1-\cos \psi)}{i u_{1}^{2}\left(1-u_{1}^{2}\right)}=\frac{t_{0}}{p_{0}} \\
t_{0}=k r_{0}(1-\cos \psi) \quad(k=\text { wave number) } \\
p_{0}=\frac{i}{2} k r_{0} u_{1}^{2}\left(1-u_{1}^{2}\right) \quad \text { (numerical distance to boundary) } \\
F\left(t_{0}\right)=\int_{0}^{t_{0}} \frac{e^{i t}}{\sqrt{2 \pi t}} d t \quad \text { (Fresnel integral) } \\
G\left(p_{0}\right)=1+i \sqrt{\pi p_{0}} e^{-p_{0}} \text { erf } \quad\left(-i \sqrt{p_{0}}\right) \quad \text { (Sommerfeld's attenuation function) }
\end{aligned}
$$

$R_{v}$ is the Fresnel reflection coefficient for vertical polarization.

When medium 2 is perfectly conducting, $u_{2}=0$ and $R_{v}^{(2)}=1$ and

$$
\begin{aligned}
A=\frac{1+R_{v}^{(1)}}{2} \cos \psi-\frac{i \sqrt{2 c}}{c+i} & \left(\frac{\sqrt{2}}{2} e^{i \frac{\pi}{4}}-F\left(t_{0}\right)\right) \\
& -\frac{e^{i t_{0}}}{(c+i) \sqrt{\pi p_{0}}}\left(1-G\left(p_{0}\right)\right) .
\end{aligned}
$$

In this particular case $\left(u_{2}=0\right)$ the solution is valid also for the groundwave mixed path where it reduces to

$$
A=\frac{i}{\sqrt{\pi p_{0}}}\left(1-G\left(p_{0}\right)\right) .
$$

This result is identical to that obtained by other authors [P. C. Clemmow, 1953; E. L. Feinberg, 1959].

From the numerical calculations [Andersen, 1962] it is seen that for small angles of $\psi$ the rate of decay of the field away from the boundary is nearly the same for the groundwave (3) and the skywave (2). This relationship is true approximately up to the end of the first Fresnel zone. One of the reasons for undertaking this study was to verify this connection between the skywave and the groundwave for an actual irregular ground.

\section{Investigated Terrain}

The terrain under investigation is situated at Reers $\varnothing$ on the west coast of Zealand, Denmark.
The horizontal profiles of the paths are shown on figures 4 and 2 respectively for the skywave and the groundwave measurement. Unfortunately, the two paths could not be made the same. The paths are nearly perpendicular to the coastline. For both the skywave path and the groundwave path there is a section of marshy ground extending from 20 to $50 \mathrm{~m}$ from the coastline; this section has a very high conductivity $\left(\sigma \sim 10^{-1} \mathrm{mho} / \mathrm{m}\right)$.

From 90 to $150 \mathrm{~m}$ from the coastline there is a field with caraway seed, representing a very low conductivity. For the groundwave mixed path there is a small hill about $100 \mathrm{~m}$ from the coastline.

It is seen that a full theoretical description should take into account the three or four different sections of ground including the variations of the profile and the different layers in the vertical direction of which nothing is known. Only the two-section problem, the sea-land case, is treated here numerically and the sea is considered infinitely conducting.

In order to investigate in more detail the properties of the ground, two groundwave measurements were made. The results are given in figure 1 . The independent variable is the distance from the transmitter which is placed $420 \mathrm{~m}$ from the coastline and the ordinate is the field strength in a $\mathrm{db}$ scale. The most marked features of the curves are the great attenuation of the caraway field and the recovery effect following it; this recovery effect is reinforced by the marshy ground close to the coast. The $15 \mathrm{Mc} / \mathrm{s}$ curve is smoother than the $25 \mathrm{Mc} / \mathrm{s}$ curve and a small recovery effect about $60 \mathrm{~m}$ from the transmitter seems to indicate a better soil. This is in agreement with an inspection of the ground. 


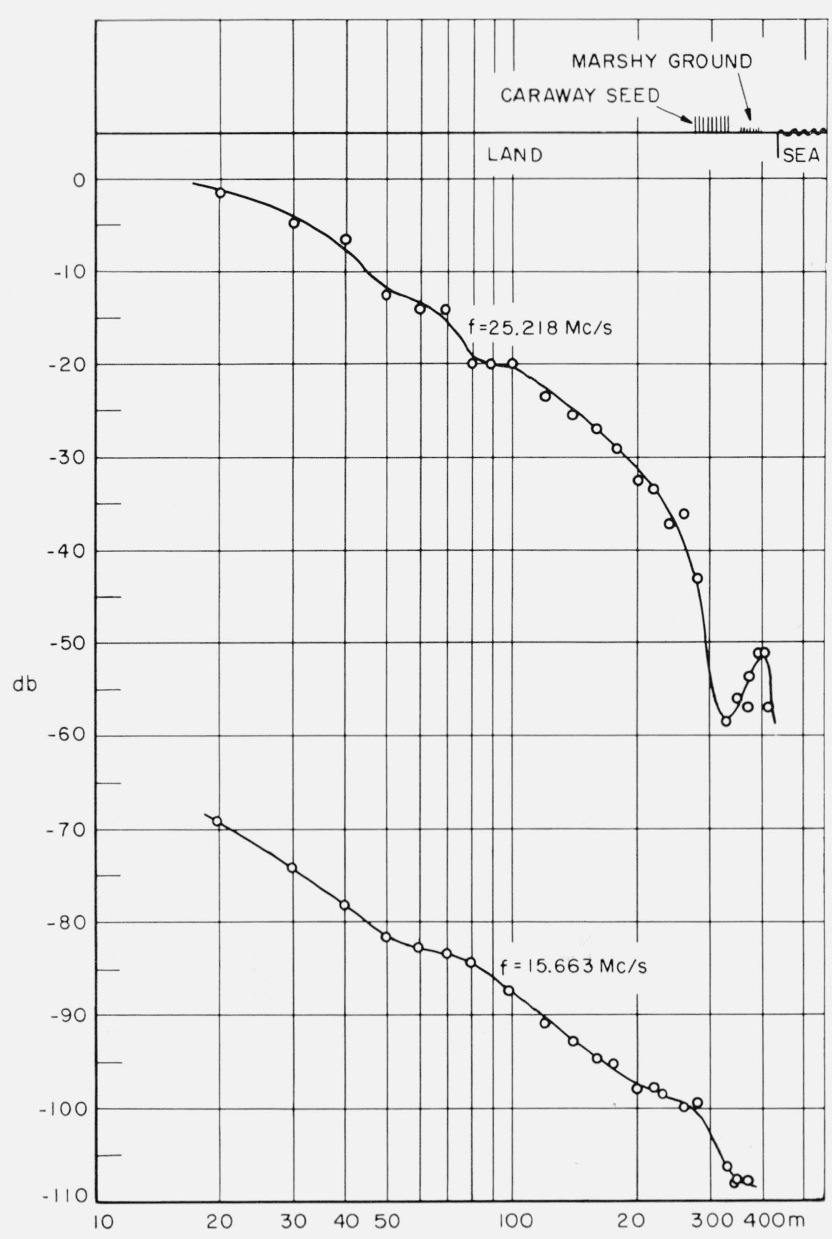

Figure 1.-Groundwave field strength over land.

\section{Groundwave Mixed-Path Measurement}

A small transmitter $(f=25.218 \mathrm{Mc} / \mathrm{s})$ was erected at Funen about $20 \mathrm{~km}$ from Reers $\varnothing$ over the water in a direction almost perpendicular to the coastline. Though there was some attenuation of the groundwave over the water this has a minor influence on the groundwave mixed path attenuation curve as measured on land; the situation is approximatively that of an incoming plane wave over a plane perfect conductor meeting a conductivity contrast when due corrections are made for the inverse distance dependence.

The result of the measurement is shown in figure 2 where the points denote the measured field strength in comparison with the theoretical curve for a groundwave transmission from a perfectly conducting ground to a lossy ground with ground constants $\epsilon=10$ and $\sigma=2.10^{-3} \mathrm{mho} / \mathrm{m}$ when the transmitter is infinitely far away. According to the theory a rapid decrease in field strength should take place right after the coastline, the so-called "converse" effect. This is seen to be true for the first two points but then a sharp increase follows when the
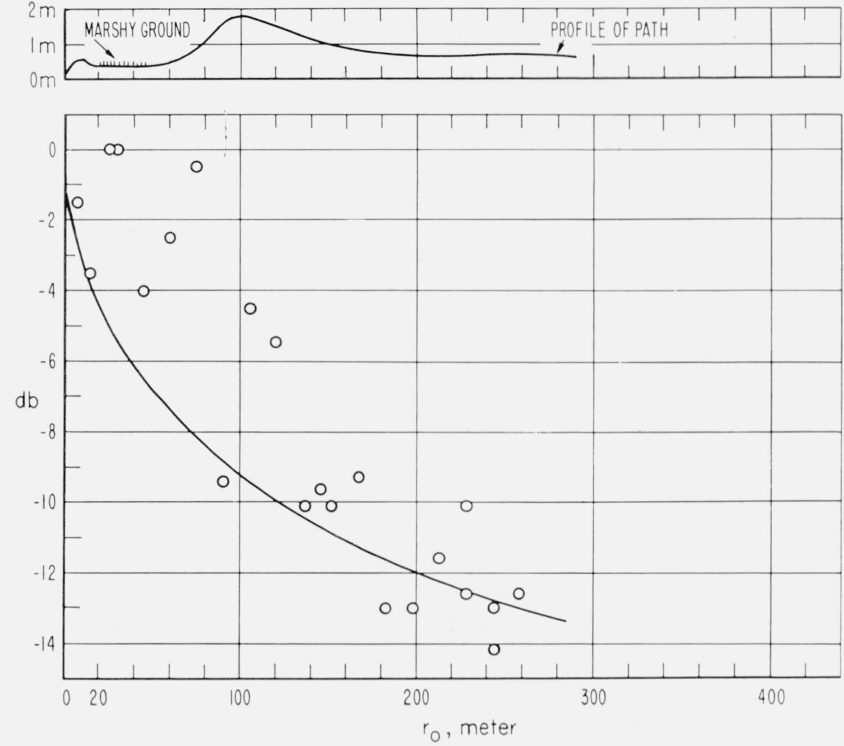

Figure 2.-Groundwave mixed path, sea-land.

O, Measured field intensity
-, Theoretical curve, $\epsilon=10, \sigma=2.10^{-3} \mathrm{mho} / \mathrm{m}$ $\mathrm{f}=25.218 \mathrm{Me} / \mathrm{s}$

high conductivity soil is entered. The small hill seems also to have some effect on the received field strength but the unknown vertical distribution of different layers in the ground may be the true cause for the rapid variation. One hundred and fifty meters from the coastline the ground is homogeneous, and the correlation between experiment and theory seems to be good.

\section{Skywave Measurement}

The experimental setup consisted of two identical receivers with identical dipole antennas and two separate recording units. The receivers were highquality communication receivers. The two sets were separated 100 to $400 \mathrm{~m}$ in different distances from the coastline. The skywave signal from a transmitter at Godthåb, Greenland, sending at a frequency of $14.447 \mathrm{Mc} / \mathrm{s}$, was recorded simultaneously at the two receiving places. Because only the relative mean field strength was desired, no absolute value was found. By calibrating the two sets to a common logarithmic scale, the mean difference between the signals at the two different places was easily found. One day's recordings were in general sufficient to provide a reliable measure. The rapidly varying component was somewhat suppressed by applying a time constant of the order of a few seconds, and the slowly varying components at the two antennas were highly correlated, because the optical path is nearly the same. The distributions of the signal have not been studied as only the mean value is of interest here. 


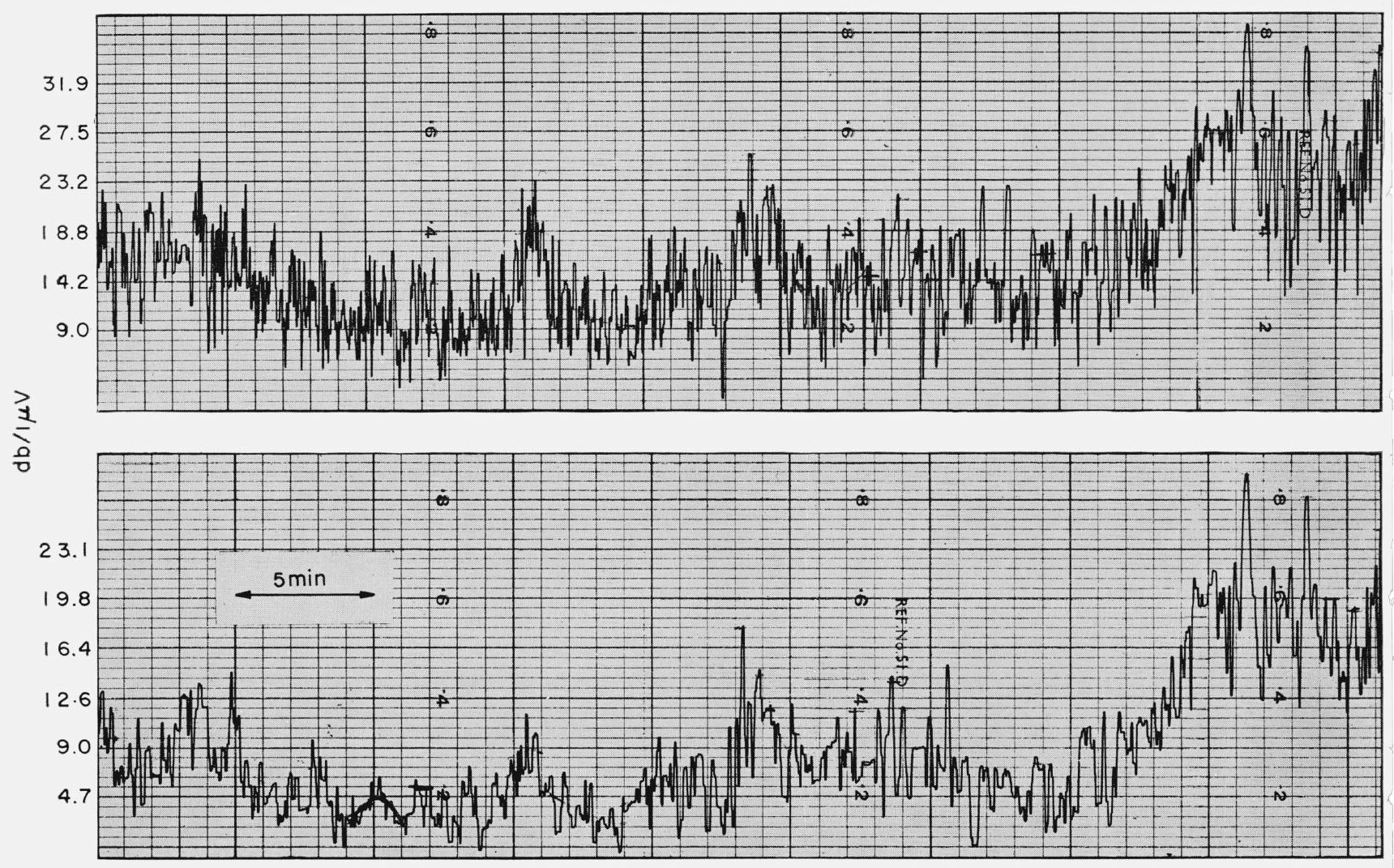

FiguRE 3.-Example of recordings.

Simultaneous recordings for $r_{0} 72 \mathrm{~m}$ (upper curve) and $r_{0} 420 \mathrm{~m}$ (lower curve) from coastline.

An example of the recordings is shown in figure 3. The upper curve is for the receiver set close to the coast $(r=72 \mathrm{~m})$, and the lower curve is for the receiver far from the coast $(r=420 \mathrm{~m})$. The mean difference between the two signals is $7.6 \mathrm{db}$. The results for other positions of the antennas are shown in figure 4 as points together with a suggested curve connecting the points Two theoretical curves are shown for two different conductivities, $\sigma=10^{-2} \mathrm{mho} / \mathrm{m}$ and $\sigma=2 \cdot 10^{-3} \mathrm{mho} / \mathrm{m}$, of which the latter should be typical according to the results from the groundwave measurement. It is seen that there is not such a good agreement in the skywave case as in the groundwave case, though the general form of the curve is correct.

The rapid fall in the beginning is present, but the high conductivity section gives an increase in field strength which displaces the curve in the vertical direction. This is similar to the recovery effect known from groundwave mixed-path theory. Furthermore, it is suggested that the caraway field gives a similar attenuation of the skywave as it does to the groundwave. Due to the inhomogeneous ground, there is a discrepancy between theory and experiment, but the form of the curve can be explained by applying the mixed path concepts known from groundwave theory, together with the theory referred to in section 2. If the ground had been

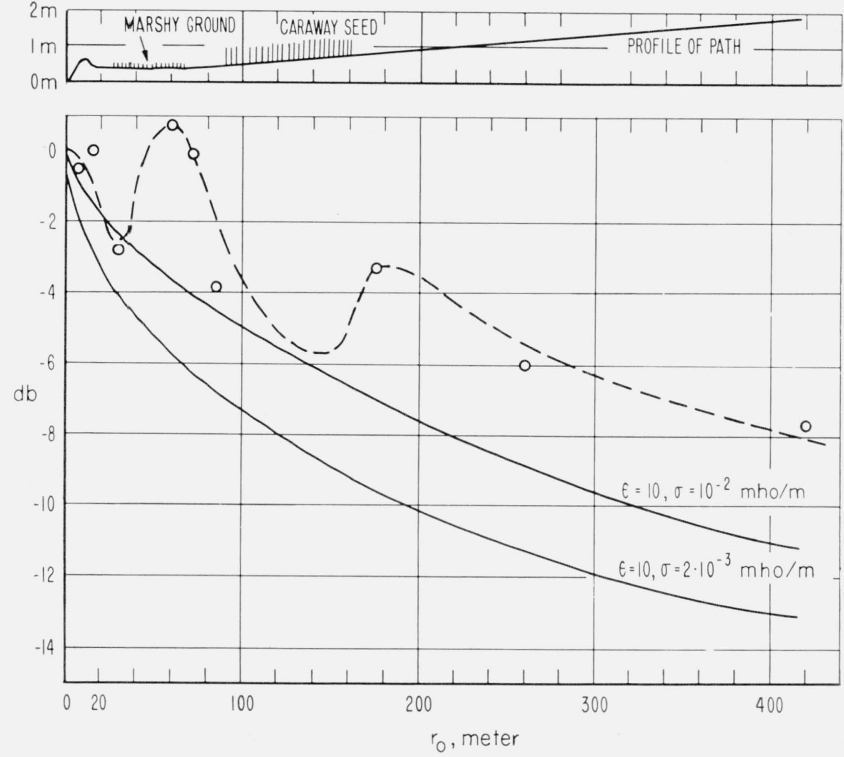

Figure 4.-Skywave measurement near a coastline.

0, Measured field intensity

- Suggested curve connecting the points $\psi=4^{0}, \mathrm{f}=14.447 \mathrm{Mc} / \mathrm{s}$
Theoretical curves 
homogeneous a loss of $13 \mathrm{db}$ could have been expected $400 \mathrm{~m}$ from the coast and even farther away, instead of the $8 \mathrm{db}$ measured.

Apart from the influence of the inhomogeneous sections, three more reasons could be given to explain some of the discrepancy. These are the finite conductivity of the sea, the diffuse reflection from the ionosphere and the curvature of the earth.

\subsection{Finite Conductivity of the Sea}

By applying formula 1 instead of formula 2 the finite conductivity of the sea would be accounted for. A rougher estimate is found by simply finding the magnitude of the proper reflection coefficient for the sea and displacing the asymptotic value $\left(r_{0} \rightarrow \infty\right)$ by an amount equal to

$$
\Delta A=20 \log \frac{1+R_{v}}{2} d b
$$

because $0 \mathrm{db}$ is referred to as the field intensity at the sea (fig. 4). The effect diminishes the coastline effect.

For the case of $\psi=4^{\circ}, \epsilon=80$ and $\sigma=4 \mathrm{mho} / \mathrm{m}$

$$
\Delta A=1.2 \mathrm{db} \text {. }
$$

\subsection{Diffuse Reflection From the Ionosphere}

It is generally believed that the vertical reception pattern and the vertical radiation pattern for an antenna are identical. When dealing with ionospheric propagation it must be remembered that the wave reflected from the ionosphere is not a plane wave but a wave distributed around some preferred direction due to the diffuse reflection from the ionosphere. Thus a sharp zero in the radiation pattern would be filled with power coming from neighboring angles, and the effective reception pattern would not have a zero. For further references see Bramley [1951] and Page and Monteath [1955].

The effect is treated here, too, because diffuse reflection will diminish the coastline effect presented in this paper. Power will enter under elevation angles greater than $4^{\circ}$, and the coastline effect is less pronounced for higher angles.

If $P(\psi)$ is the angular distribution of incoming power distributed around some preferred direction $\psi_{0}$ and $A(\psi)$ is the vertical radiation pattern (power), then an effective reception pattern can be defined as

$$
B_{0}\left(\psi_{0}\right)=\int P(\psi) \cdot A(\psi) d \psi
$$

A usual assumption is that the power distribution is normal

$$
\begin{aligned}
P(\psi) & =\frac{P_{0}}{\sqrt{2 \pi \theta_{0}}} e^{-\frac{\theta^{2}}{2 \theta_{0}^{2}}} \quad\left(P_{0} \text { total power }\right) \\
& =\psi_{0}+\theta .
\end{aligned}
$$

$\theta_{0}$ is the standard deviation of the distribution. It can easily be shown that if

$$
A(\psi)=A\left(\psi_{0}\right) \cdot\left[1+a_{1} \theta+a_{2} \theta^{2}+\ldots . .\right]
$$

then

$$
B_{0}\left(\psi_{0}\right)=P_{0} \cdot A\left(\psi_{0}\right) \cdot\left[1+a_{2} \theta_{0}^{2}+\text { higher order terms }\right] .
$$

Assuming $A(\psi)=\left(\frac{1+R_{v}}{2} \cos \psi\right)^{2}$, the radiation pattern for a vertical dipole over lossy ground, then

$$
\begin{aligned}
a_{2}=\cot \psi_{0} & -\operatorname{tg} \psi_{0}-\frac{\cos \psi_{0}}{\sin \psi_{0}+u} \\
& -\left[4+\frac{2 \cos \psi_{0} \cot \psi_{0}-3 \sin \psi_{0}}{\sin \psi_{0}+u}-\frac{2 \cos ^{2} \psi_{0}}{\left(\sin \psi_{0}+u\right)^{2}}\right]
\end{aligned}
$$

Thus the influence of the diffuseness of the ionosphere is different for an antenna on the sea $(u=0)$ and on the ground $(u \neq 0)$. The effect turns out to be small though as can be seen from table $1, B$ is the ratio

$$
\frac{\left(1+a_{2} \theta_{0}^{2}\right)_{u=0}}{\left(1+a_{2} \theta_{0}^{2}\right)_{u \neq 0}}
$$

measured in $\mathrm{db}, \psi_{0}=4^{0}, \sigma=2 \cdot 10^{-3} \mathrm{mho} / \mathrm{m}, \epsilon=10$, $f=15 \mathrm{Mc} / \mathrm{s}$. The effect diminishes the coastline effect.

\begin{tabular}{c|c|c|c|c|c} 
TABLE 1 \\
\hline \hline$\theta_{0}^{\circ}$ & $1^{\circ}$ & $2^{\circ}$ & $3^{\circ}$ & $4^{\circ}$ & $5^{\circ}$ \\
\hline B db & 0.1 & 0.4 & 0.8 & 1.3 & 1.9 \\
\hline
\end{tabular}

$\theta_{0}$ could be computed if the distribution of the difference between the signals arriving at the spaced antennas was known [Bramley, 1951]. This has not been done here. Bramley reports $\theta_{0}$ around 1 to $2^{\circ}$ for vertical incidence first order reflections, and Page and Monteath report $\theta_{0}$ of the order of $10^{\circ}$ for a lower frequency. Some observations have also been made on second order reflections. These show much wider angular distributions and indicate that the intermediate ground reflection may give rise to a greater spread in the direction of arrival than that produced by the ionosphere.

For such a low angle of arrival as used here $\left(\psi_{0} \approx 4^{\circ}\right)$, the distribution of the power is probably somewhat skew so more power is concentrated at higher angles; this effect would also increase $B$.

\subsection{Effect of Curvature of Earth}

For small angles of arrival the plane earth assumption is no longer valid. The field pattern based on the Fresnel reflection coefficient has a zero for $\psi=0$, but the solution for a curved lossy surface has a finite value. Numerical results covering all practical cases have been given by Wait and Conda [1958], and the mixed path over spherical earth has also been treated by Wait [1961]. 
In our case $\psi=4^{\circ}$, and it turns out that for $f=15$ $\mathrm{Mc} / \mathrm{s}$ the curvature of the earth can be neglected. The geometrical optics approximation is valid, and the result for the curved earth is the same as for a plane earth. For $\psi<1 / 2^{\circ}$ Wait's results should be applied.

\section{Conclusion}

The skywave field strength from a distant transmitter has been measured near a coastline in order to find the rate of attenuation when passing from a well conducting (perfectly conducting) medium to a lossy medium. For small angles of arrival much power is lost in the ground so it is important to know how close to the coast one should be in order to utilize the well conducting sea water. Previous theoretical work suggested that when the coastline was in the first Fresnel zone in relation to the receiver, the rate of decay of the groundwave and the skywave were nearly the same. This has partly been verified here and suggests that a mixed-path groundwave measurement should give valuable information about the ground when a specific ground has to be evaluated for HF transmitting or receiving purposes.

For the ground studied here it turns out that there is some disagreement with simple two-medium theory due to inhomogeneities of the ground. A well conducting section near the coastline has the effect of displacing the "effective" coastline, the field strength does not decay as rapidly as expected, but the effect is the same on the groundwave and the skywave. The ground consists of several sections of different conductivities, and they give rise to recovery effects and converse-effects. These concepts are easily extended to the skywave in order to explain the deviations from theory in a qualitative manner.

It is shown that the effects of finite conductivity of the sea, diffuse reflection from the ionosphere, and curvature of the earth are of minor importance. The first two may give rise to a decrease in the above mentioned coastline effect by a total amount of $3 \mathrm{db}$.
This study has been supported by Air Force Cambridge Research Center of the Air Research and Development Command, United States Air Force, through its European Office, under Contract No. AF 61(052)-503. The Danish Post and Telegraphs Administration furnished the author with the necessary experimental apparatus as well as instructions and help in how to use it. The author expresses his gratitude to these organizations.

\section{References}

Andersen, J. Bach (June 1962), The radiation field from a vertical dipole on an inhomogeneous ground, Proc. Symposium on Electromagnetic Theory and Antennas, Copenhagen (in press).

Bramley, E. N. (Jan. 1951), Diversity effects in spaced aerial reception of ionospheric waves, Proc. Inst. Elec. Engrs 98, pt 3, 19-25.

Clemmow, P. C. (1953), Radio propagation over a flat earth across a boundary separating two different media, Phil. Trans. Roy. Soc. London A246, 1-55.

Feinberg, E. L. (1959), Propagation of radio waves along an inhomogeneous surface, Nuovo Cimento, Suppl. 11, Serie $x$.

Page, H., and G. D. Monteath (May 1955), The vertical radiation patterns of medium wave broadcasting aerials, Proc. Inst. Elec. Engrs. B10\%, No. 3, 279-296.

Wait, J. R., and A. M. Conda (Oct. 1958), Pattern of an antenna on a curved lossy surface, IRE Trans. on Ant. Prop. AP 6, No. 4, 348-359.

Wait, J. R. (1961), On the theory of mixed path groundwave propagation over a spherical earth, J. Res. NBS 65D (Radio Prop.) No. 4, 401-410.

Wilson, A. C. (1961) Measurements of low angle radiation from a monopole, J. Res. NBS 65D (Radio Prop.) No. 6, $641-645$. 